\title{
Le fardeau du trouble d'anxiété généralisée au Canada
}

\section{Louise Pelletier, M. D. (1); Siobhan O'Donnell, M. Sc. (1); Louise McRae, B. Sc. (1); Jean Grenier, Ph. D. (2,3)}

Cet article a fait l'objet d'une évaluation par les pairs.

Diffuser cet article sur Twitter

\section{Résumé}

Introduction : Bien que le trouble d'anxiété généralisée (TAG) soit une affection courante et incapacitante, peu d'études ont été menées à son sujet au Canada. Nous avons comparé les Canadiens atteints du TAG avec ceux atteints de dépression sur le plan de leurs caractéristiques, de leur état de santé, de leur utilisation des services de santé et de leurs besoins en matière de santé.

Méthodologie : Les données sont tirées de l'Enquête sur la santé dans les collectivités canadiennes - Santé mentale de 2012, qui portait sur un échantillon représentatif de la population canadienne de 15 ans et plus ( $\mathrm{n}=23$ 709; taux de réponse de 68,9\%). Nous avons sélectionné les répondants ayant fait état de symptômes compatibles avec un TAG ou un épisode dépressif majeur (EDM) dans les 12 mois précédant l'enquête ( $\mathrm{n}=1598$ ). Les estimations ont été pondérées afin de représenter la population des ménages au Canada. Nous avons mené des analyses de régression logistiques multidimensionnelles multinomiales et descriptives.

Résultats : En 2012, environ 700000 (2,5 \%) Canadiens de 15 ans et plus ont fait état de symptômes compatibles avec un TAG dans les 12 derniers mois. La moitié d'entre eux ont également mentionné des symptômes d'EDM. La fréquence des perceptions d'une santé passable ou mauvaise $(29,7 \%)$, de détresse psychologique modérée et sévère $(81,2 \%)$ et d'incapacité modérée et sévère $(28,1 \%)$ était comparable (voire légèrement supérieure) chez les personnes présentant un TAG seulement par rapport aux personnes présentant un EDM seulement (respectivement 24,7 \%, 78,8 \% et 24,8 \%). Les pires résultats de santé ont été observés chez les personnes souffrant d'un TAG et d'un EDM en comorbidité : 47,3 \% ont fait état d'une santé passable ou mauvaise, 94,0 \% d'une détresse psychologique modérée ou sévère et 52,4 \% d'une incapacité modérée ou sévère.

Près de $50 \%$ des personnes souffrant d'un TAG et d'un EDM en comorbidité ont déclaré que leurs besoins en matière de santé n'avaient pas été satisfaits ou avaient été partiellement satisfaits, contre environ $30 \%$ des personnes souffrant d'un TAG ou d'un EDM seulement.

Conclusion : Bien que le TAG soit associé à des degrés de détresse et d'incapacité comparables (ou légèrement supérieurs) à l'EDM, l'état de santé des personnes présentant ces deux affections en même temps est nettement moins bon que celui des personnes présentant uniquement l'un ou l'autre. Il est essentiel d'améliorer le diagnostic, le dépistage des comorbidités et la prise en charge pour réduire les impacts de ce trouble mental.

Mots-clés : trouble d'anxiété généralisée, impact, prévalence, incapacité, Canada, épisode dépressif majeur

\section{Introduction}

En 2012, environ 2,4 millions $(8,7 \%)$ de Canadiens de 15 ans et plus ont fait état de symptômes compatibles avec un trouble d'anxiété généralisée (TAG) au cours de leur vie. Parmi eux, 30 \% (soit 2,6 \% de la population canadienne) ont fait état de ces

\section{Points saillants}

- En 2012, environ 700000 (2,5 \%) Canadiens de 15 ans et plus ont fait état de symptômes compatibles avec un trouble d'anxiété généralisée (TAG) dans les 12 mois précédant l'enquête. La moitié d'entre eux ont aussi fait état de symptômes compatibles avec un épisode dépressif majeur (EDM). Ces estimations sont conservatrices, car il se peut que les personnes atteintes de ces affections, particulièrement celles aux prises avec des symptômes graves, soient réticentes à participer à une enquête sur la santé.

- Bien que les personnes présentant un TAG aient fait état d'une santé mentale passable ou mauvaise, de détresse psychologique et d'incapacité de manière similaire à celles souffrant d'un EDM, les résultats de santé étaient nettement moins bons dans leur ensemble chez les personnes présentant les deux affections en comorbidité.

- Près de $50 \%$ des personnes présentant un TAG et un EDM en comorbidité ont déclaré que leurs besoins en matière de santé mentale n'avaient pas été satisfaits ou avaient été partiellement satisfaits, contre environ $30 \%$ des personnes présentant un TAG ou un EDM seulement.

symptômes dans les 12 mois ayant précédé l'enquête' ${ }^{1}$. Cette enquête était la première à fournir des estimations du TAG à l'échelle de la population canadienne. Des études épidémiologiques menées aux États-Unis, en Europe et en Australie à l'aide d'une méthodologie comparable ont produit des estimations semblables de la prévalence sur 12 mois (de 1 à $4 \%)^{2,3,4}$.

\section{Rattachement des auteurs :}

1. Centre de prévention des maladies chroniques, Agence de la santé publique du Canada, Ottawa (Ontario), Canada

2. Institut de recherche de l'Hôpital Montfort, Centre de recherche C.T. Lamont en soins de santé primaires, Ottawa (Ontario), Canada

3. Département de médecine familiale, Université d'Ottawa, Ottawa (Ontario), Canada

Correspondance : Louise Pelletier, Centre de prévention des maladies chroniques, Agence de la santé publique du Canada, 785, avenue Carling, IA : 6806A, Ottawa (Ontario) K1A 0K9; tél. : 613-960-5339; courriel : louise.pelletier@phac-aspc.gc.ca 
Sur le plan clinique, les personnes présentant un TAG souffrent d'anxiété excessive et s'inquiètent à propos de toutes sortes de sujets (p. ex. école, travail, relations interpersonnelles), des événements de leur vie et de leurs activités quotidiennes. Cette inquiétude est présente dans la majorité des journées, dure au moins 6 mois, et elle est clairement excessive et difficile à maîtriser $^{5,6}$. Pour permettre l'établissement du diagnostic de TAG, les symptômes d'anxiété et d'inquiétude excessives doivent être accompagnés d'au moins trois des six symptômes suivants chez l'adulte et d'au moins l'un de ces symptômes chez l'enfant : agitation, fatigue, difficulté à se concentrer, irritabilité, tension musculaire et troubles du sommeil. L'anxiété, l'inquiétude et les symptômes physiques causent une détresse significative sur le plan clinique et affectent des volets importants du fonctionnement quotidien, et la perturbation n'est pas attribuable aux effets physiologiques d'une substance ou d'un autre problème de santé ${ }^{6}$

Le TAG débute généralement à la fin de l'adolescence ou dans la vingtaine, mais il peut survenir à n'importe quelle période de la vie ${ }^{7}$. Plusieurs adultes atteints d'un TAG disent qu'ils ont été anxieux presque toute leur vie. Comme l'anxiété et l'inquiétude excessives sont mal définies et qu'elles apparaissent généralement de façon insidieuse chez les jeunes, il faut habituellement plus de 10 ans pour poser un diagnostic $^{7}$. Inversement, les personnes plus âgées, chez qui le TAG est généralement lié à un évènement stressant de la vie, sont plus nombreuses à consulter un professionnel de la santé durant la première année des symptômes ${ }^{7}$. Le TAG est considéré depuis longtemps comme une affection chronique dont les symptômes vont en augmentant et en diminuant. Cependant, quelques études longitudinales de cohorte ont révélé que jusqu'à $50 \%$ des personnes souffrant de TAG pouvaient passer de longues périodes sans symptômes, et quoiqu'un certain nombre d'entre elles fassent une rechute ${ }^{8,9,10}$. Les incapacités sont plus fréquentes chez les personnes dont les symptômes se manifestent à un plus jeune âge ou sont traités tardivement ${ }^{10,11}$. La présence de comorbidités contribue aussi à la détérioration de l'état de santé6,8.

L'identification précoce du TAG est en grande partie compliquée par le fait que les individus affectés sont peu nombreux à consulter un professionnel de la santé expressément en raison d'une anxiété ou d'une inquiétude excessive ${ }^{12}$. Leurs consultations sont plutôt motivées par des symptômes somatiques comme la fatigue, des troubles du sommeil, des maux de tête, des symptômes gastro-intestinaux et des symptômes associés à des comorbidités ${ }^{5}$.

Les personnes présentant un TAG dont les symptômes sont légers peuvent acquérir de bons mécanismes d'adaptation, si bien que leur affection perturbera peu leurs activités quotidiennes. Dans les cas les plus graves, le fonctionnement de la personne peut toutefois être gravement altéré?.

Le TAG est fréquemment accompagné d'autres problèmes de santé mentale, ce qui en complique aussi le diagnostic et le traitement. Selon certaines études, jusqu'à $90 \%$ des personnes présentant un TAG ont des troubles mentaux comorbides au cours de leur vie, notamment la dépression et d'autres troubles d'anxiétét,12. Les symptômes du TAG peuvent aussi être concomitants à des problèmes de santé physique comme des douleurs chroniques, le diabète et une cardiopathie et, du même coup, exacerber ces affections ou affecter la capacité de la personne à les gérer ${ }^{13}$.

Avant les dix dernières années, le TAG n'était pas reconnu comme une maladie à part entière car les professionnels de la santé constataient sa présence très souvent en association avec d'autres problèmes de santé mentale, particulièrement la dépression. Cependant, un certain nombre d'études ont permis de réfuter cette croyance ${ }^{7,14}$. Ces études ont aussi montré que le degré d'incapacité des personnes présentant un TAG « pur » équivalait à celui observé dans les cas de dépression majeure "pure » ou d'autres problèmes de santé physique ou mentale gravement incapacitants ${ }^{7,15,16}$. Parmi les caractéristiques essentielles du TAG, notons que les personnes touchées demandent rarement de l'aide pour leurs symptômes d'anxiété, mais qu'elles utilisent beaucoup les ressources en santé et qu'elles sont présentes dans une proportion démesurément élevée dans les consultations en soins de santé4,16,17.

Bien que la dépression ait été abondamment étudiée, les études épidémiologiques sur le TAG sont peu nombreuses au Canada. Notre étude, qui porte sur les données concernant un échantillon conçu pour être représentatif de la population canadienne de 15 ans et plus, vise à : 1) comparer les personnes ayant eu des symptômes compatibles avec le TAG (avec ou sans EDM en comorbidité) dans les 12 derniers mois et les personnes ayant uniquement eu un EDM, sur le plan des caractéristiques sociodémographiques, comportementales et de santé, 2) comparer les personnes présentant un TAG (avec ou sans EDM en comorbidité) aux personnes souffrant uniquement d'un EDM sur le plan de l'état de santé, de l'utilisation des services de santé et des besoins en matière de santé et 3) déterminer s'il existe des différences en fonction de l'âge dans les associations entre, d'une part, l'état de santé ou l'utilisation des services de santé et, d'autre part, ces problèmes de santé mentale.

\section{Méthodologie}

\section{Source des données et échantillon}

L'Enquête sur la santé dans les collectivités canadiennes - Santé mentale de 2012 (ESCC-SM) est une enquête transversale fondée sur un plan d'échantillonnage en grappes stratifié à plusieurs degrés qui porte sur la population de 15 ans et plus des 10 provinces canadiennes ${ }^{18}$. Les personnes suivantes ont été exclues de l'enquête : celles vivant sur des réserves et les terres de la Couronne, les sans-abri, les membres à temps plein des Forces canadiennes et les personnes vivant en établissement, soit au total environ $3 \%$ de la population cible.

L'ESCC-SM visait à collecter des données sur la santé mentale, sur l'accès aux services et soutiens formels et informels en santé mentale et sur le besoin perçu de ces services et soutiens, sur le fonctionnement général et l'incapacité, sur les déterminants de la santé et sur les caractéristiques sociodémographiques de la population.

Le taux de réponse global à l'ESCC-SM a été de 68,9\%. Pour les besoins de notre étude, nous avons utilisé le fichier partagé ( $n=23$ 709) et exclu les répondants ( $\mathrm{n}=293$ ) pour lesquels les mesures fondées sur les symptômes de TAG et d'EDM étaient incomplètes. Notre échantillon comportait donc au total 23416 répondants.

De plus amples renseignements sur l'ESCC-SM, dont le questionnaire, sont disponibles à l'adresse : http://www23.statcan .gc.ca/imdb/p2SV_f.pl?Function = getSurvey $\&$ SDDS $=5015 \&$ Item_Id $=119790$ \&lang $=$ en 


\section{Mesures fondées sur les symptômes de TAG et d'EDM}

Les critères de l'ESCC-SM et de la Composite International Diagnostic Interview (CIDI, Entrevue composite diagnostique internationale) de l'Organisation mondiale de la santé constituent une version modifiée du questionnaire CIDI original, qui est un instrument normalisé d'évaluation des troubles mentaux fondé sur l'opérationnalisation des définitions et des critères du Manuel diagnostique et statistique des troubles mentaux, $4^{e}$ édition (DSM-IV) ${ }^{19,20}$. Le questionnaire CIDI, conçu pour mesurer la prévalence des troubles mentaux à l'échelle des collectivités, peut être administré par des enquêteurs non spécialisés.

Les répondants ont été considérés comme présentant des symptômes compatibles avec le TAG s'ils avaient satisfait aux critères de l'ESCC-SM et de la CIDI dans les 12 derniers mois $^{18}$. Une méthode semblable a été utilisée pour sélectionner les répondants ayant présenté des symptômes compatibles avec l'EDM dans les 12 derniers mois. L'encadré 1 contient de plus amples renseignements à ce sujet.

\section{Caractéristiques sociodémographiques}

Nous avons étudié les caractéristiques sociodémographiques suivantes : sexe (féminin ou masculin), âge (de 15 à 29 ans, de 30 à 49 ans, 50 ans et plus et âge moyen), état matrimonial (célibataire, divorcé/séparé/ veuf, marié/conjoint de fait), plus haut niveau de scolarité atteint par le répondant (études secondaires partielles, diplôme d'études secondaires, études postsecondaires partielles, diplôme d'études postsecondaires), quintile de revenu du ménage ajusté, activité professionnelle dans la dernière semaine (étudiant, sans emploi dont incapacité permanente et retraite, en activité), immigrant (oui, non), statut d'Autochtone (oui, non), et milieu (urbain, rural).

Pour déterminer le quintile de revenu du ménage ajusté, nous avons réparti les répondants en quintiles fondés sur le rapport ajusté entre le revenu total de leur ménage et le seuil de faible revenu correspondant à la taille de leur ménage et de leur collectivité, comme défini par Statistique Canada ${ }^{21}$.

\section{Caractéristiques comportementales et de santé}

Les catégories de tabagisme utilisées étaient : " fumeur quotidien », « fumeur occasionnel ou ex-fumeur » et « non-fumeur ». Le nombre de comorbidités physiques était fondé sur la mention par les répondants de diagnostics d'asthme, de maladie pulmonaire obstructive chronique, d'arthrite, de problèmes de dos, de maladie de Crohn, de colite ulcéreuse, de diabète, d'épilepsie, de cardiopathie, de cancer, d'accident vasculaire cérébral, de maladie d'Alzheimer et de toute autre démence établis par un professionnel de la santé. Chaque affection comptait comme une comorbidité physique distincte et nous avons utilisé les trois catégories « aucune », « 1 ou 2 » et « 3 et plus ».

Les répondants ont été considérés comme présentant des symptômes compatibles avec un trouble de consommation de substances s'ils avaient satisfait aux critères de dépendance ou d'abus d'alcool ou de drogues de l'ESCC-SM et de la CIDI dans les 12 derniers mois (encadré 1).

\section{État de santé}

L'état de santé perçu est un indicateur de l'état de santé global. Pour le mesurer, l'enquête comportait la question suivante : " En général, diriez-vous que votre santé est excellente, très bonne, bonne, passable ou mauvaise? » Les répondants ayant déclaré que leur santé était " passable » ou " mauvaise » ont été classés comme ayant un état de santé sous-optimal.

Pour mesurer la santé mentale autoévaluée, l'enquête comportait la question suivante : « En général, diriez-vous que votre santé mentale est excellente, très bonne, bonne, passable ou mauvaise? » Les répondants ayant déclaré que leur santé mentale était « passable » ou « mauvaise » ont été classés comme ayant un état de santé mentale sous-optimal.

Le degré de détresse psychologique durant le dernier mois a été mesuré à l'aide du score à l'échelle de Kessler à 6 énoncés (K6), qui évalue dans quelle mesure la personne s'est sentie "nerveuse », " désespérée ", " agitée ou ne tenant pas en place », " si déprimée que plus rien ne pouvait la réconforter », " que tout était un effort » et « bonne à rien » durant le dernier mois ${ }^{22}$. Le score, qui pouvait aller de 0 à 24 , a été classé en trois catégories : " aucune détresse psychologique », " détresse psychologique modérée " et " détresse psychologique sévère $»^{23}$.

Le degré d'incapacité dans les 30 derniers jours a été déterminé à l'aide du WHO
Disability Assessment Schedule 2.0 (WHODAS; Plan d'évaluation des invalidités de l'Organisation mondiale de la santé), qui porte sur six volets de l'incapacité : les fonctions cognitives, la mobilité, les soins personnels, les rapports avec les autres, les activités de la vie quotidienne et la participation. Le score global, qui pouvait aller de 0 (aucune incapacité) à 100 (incapacité complète), a été attribué conformément à la méthode décrite dans le manuel WHODAS $2.0^{24}$. Les catégories d'incapacité étaient " aucune incapacité ", " incapacité légère ", " incapacité modérée » et "incapacité sévère ou extrême $»^{25}$.

\section{Consultations médicales et besoin perçu de soins de santé mentale}

Pour déterminer si les répondants avaient consulté un professionnel de la santé pour des problèmes de santé mentale dans les 12 derniers mois, on leur a demandé s'ils avaient eu une rencontre ou une discussion téléphonique avec au moins un des professionnels suivants en raison de difficultés émotionnelles, de troubles de santé mentale ou de consommation d'alcool ou de drogues durant les 12 derniers mois : psychiatre; psychologue; médecin de famille ou praticien général; infirmière; travailleur social, conseiller ou psychothérapeute. Les répondants ayant répondu " oui » dans le cas d'un psychiatre, d'un psychologue, d'un travailleur social, d'un conseiller ou d'un psychothérapeute ont été considérés comme ayant consulté un professionnel de la santé mentale dans les 12 derniers mois.

Le besoin perçu de soins de santé mentale dans les 12 derniers mois a été déterminé en répartissant les répondants en deux catégories, selon s'ils avaient mentionné un besoin (p. ex. information, médicaments, counseling) ou non. Dans l'affirmative, on a déterminé si le besoin avait été satisfait totalement, partiellement ou pas du tout.

\section{Analyse statistique}

Pour tenir compte de la répartition de l'échantillon et de la conception de l'enquête, nous avons pondéré toutes les estimations en fonction de la pondération utilisée par Statistique Canada, de manière à ce que les résultats représentent la population des ménages de 15 ans et plus dans les 10 provinces canadiennes en $2012^{18}$. Des estimations de la variance (intervalles de confiance à $95 \%$ et coefficients de 


\section{ENCADRÉ 1}

Critères de TAG, d'EDM et de trouble de consommation de substances de l'ESCC-SM et de la CIDI ${ }^{21}$

L'Enquête sur la santé dans les collectivités canadiennes - Santé mentale de 2012 (ESCC-SM) repose sur une version modifiée du questionnaire Composite International Diagnostic Interview 3.0 (CIDI) de l'Organisation mondiale de la santé pour la classification des personnes atteintes de troubles de santé mentale ou de consommation de substances. Bien qu'il ne permette pas de poser un diagnostic clinique, cet instrument normalisé est couramment utilisé pour évaluer les troubles mentaux correspondant aux critères du Manuel diagnostique et statistique des troubles mentaux, $4^{e}$ édition (DSM-IV) dans les enquêtes sur la population.

\section{Trouble d'anxiété généralisée (TAG)}

Pour les besoins de l'enquête, les répondants obéissant aux critères suivants de l'ESCC-SM et de la CIDI d'un trouble d'anxiété généralisée au cours de la vie sont ceux qui ont mentionné :

- une anxiété et une inquiétude excessives et une anxiété liée à au moins un événement ou une activité durant au moins six mois;

- une difficulté à contrôler l'inquiétude;

- que l'anxiété et l'inquiétude étaient associées à au moins trois symptômes d'anxiété;

- une détresse cliniquement significative ou une altération importante du fonctionnement social, professionnel ou autre causée par l'anxiété, l'inquiétude ou des symptômes physiques.

Pour les besoins de l'enquête, les répondants obéissant aux critères suivants de l'ESCC-SM et de la CIDI d'un trouble d'anxiété généralisée dans les 12 derniers mois sont ceux qui ont mentionné :

- avoir satisfait aux critères du trouble d'anxiété généralisée au cours de la vie;

- avoir vécu un épisode d'anxiété généralisée durant au moins 6 mois dans les 12 mois ayant précédé l'enquête;

- une détresse cliniquement significative ou une altération de leur fonctionnement social, professionnel ou autre.

\section{Épisode dépressif majeur (EDM)}

Pour les besoins de l'enquête, les répondants obéissant aux critères suivants de l’ESCC-SM et de la CIDI d'un épisode dépressif majeur au cours de la vie sont ceux qui ont mentionné :

- une période de deux semaines ou plus d'humeur dépressive ou de perte d'intérêt ou de plaisir ET au moins cinq autres symptômes;

- une détresse cliniquement significative ou une altération du fonctionnement social ou professionnel;

- que les symptômes ne sont pas explicables par un deuil.

Pour les besoins de l'enquête, les répondants obéissant aux critères suivants de l'ESCC-SM et de la CIDI d'un épisode dépressif majeur dans les 12 derniers mois sont ceux qui :

- satisfont aux critères diagnostiques d'un épisode dépressif majeur au cours de la vie (voir ci-dessus);

- ont fait état d'un épisode dépressif majeur dans les 12 derniers mois;

- ont fait état d'une altération marquée de leur fonctionnement professionnel ou social.

\section{Tout trouble de consommation de substances (dépendance ou abus) au cours des 12 derniers mois}

Le paramètre « Tout trouble de consommation de substances (dépendance ou abus) durant les 12 derniers mois » englobe la consommation d'alcool, de cannabis, de cocaïne, de drogues récréatives, d'hallucinogènes, d'héroïne, d'opium, de produits à inhaler et de solvants, ainsi que l'utilisation de stimulants et d'analgésiques à des fins non médicales. Les répondants qui remplissent les critères de dépendance ou d'abus d'alcool ou de drogues durant les 12 derniers mois ont été classés comme ayant des symptômes compatibles avec un trouble de consommation de substances.

- La dépendance est caractérisée par un mode récurrent de consommation où au moins trois des symptômes suivants se manifestent dans les 12 mois : tolérance accrue; sevrage; consommation accrue; tentatives de cessation infructueuses; beaucoup de temps perdu en raison de la récupération et de la consommation; diminution des activités et poursuite de la consommation malgré les problèmes physiques et psychologiques persistants causés ou exacerbés par la substance.

- L'abus est caractérisé par un mode récurrent de consommation où au moins un des symptômes suivants se manifeste : impossibilité de remplir d'importantes obligations au travail, à l'école ou à la maison; consommation dans des situations où il est dangereux de le faire; problèmes récurrents liés à l'alcool et poursuite de la consommation malgré les problèmes sociaux et interpersonnels causés ou exacerbés par l'alcool. Par définition, les répondants qui remplissent les critères de dépendance ne répondent pas aux critères d'abus. 
variation) ont été établies à l'aide des poids bootstrap fournis avec les données ${ }^{26}$.

Nous avons effectué des analyses descriptives de tableaux croisés pour décrire les répondants ayant fait état de symptômes compatibles avec le TAG seulement, le TAG et l'EDM en comorbidité, et l'EDM seulement dans les 12 mois ayant précédé l'enquête. Le test du chi carré de Rao-Scott visant à vérifier la qualité de l'ajustement et une analyse de régression linéaire ont été utilisés pour examiner la relation entre les variables nominales et continues (p. ex. âge moyen), respectivement, et les sousgroupes mentionnés ci-dessus. Le seuil de signification statistique a été fixé à $p<0,01$.

Nous avons mené une analyse de régression logistique multidimensionnelle multinomiale pour comparer l'état de santé, les consultations médicales et le besoin perçu en soins de santé mentale chez les répondants ayant fait état de symptômes compatibles avec le TAG seulement, le TAG et l'EDM en comorbidité et l'EDM seulement dans les 12 derniers mois. Les covariables choisies, sur la base de la littérature sur le sujet, ont été le sexe, l'âge, l'état matrimonial, le niveau de scolarité, le quintile de revenu du ménage, l'activité professionnelle, l'immigration, le statut d'Autochtone, le tabagisme, les troubles de consommation d'alcool et de drogues et les comorbidités physiques ${ }^{27,28}$.

Nous avons aussi vérifié les interactions entre l'âge et les divers résultats sur le plan de la santé et de l'utilisation des services de santé, après ajustement en fonction des variables sociodémographiques et de santé, pour les troubles mentaux étudiés (TAG seulement, TAG et EDM en comorbidité et EDM seulement). Des modèles statistiquement significatifs ayant une valeur $p<0,01$ ont été établis.

Nous avons réalisé les analyses à l'aide du logiciel SAS Enterprise Guide version 5.1 (SAS Institute, Cary, Caroline du Nord, États-Unis).

\section{Résultats}

\section{Prévalence et caractéristiques sociodémo- graphiques, comportementales et de santé}

La prévalence des symptômes compatibles avec un TAG dans les 12 derniers mois était de $2,5 \%$ dans la population canadienne de 15 ans et plus, la moitié de ces personnes ayant aussi fait état de symptômes compatibles avec un EDM (tableau 1).

La relation entre les troubles étudiés (TAG seulement, TAG et EDM en comorbidité et EDM seulement) et les caractéristiques sociodémographiques, comportementales et de santé était statistiquement significative uniquement pour l'âge et pour le nombre de comorbidités physiques (tableau 1).

Comparativement aux personnes présentant un EDM seulement, les personnes présentant un TAG seulement étaient plus âgées (43,8 ans contre 38,0 ans en moyenne) et plus nombreuses à avoir au moins une comorbidité physique $(65,9 \%$ contre $49,0 \%)$. Cette dernière observation s'explique vraisemblablement par un effet de confusion attribuable à l'âge. De même, comparativement aux personnes présentant seulement un EDM, les personnes présentant un TAG et un EDM en comorbidité étaient

TABLEAU 1

Caractéristiques sociodémographiques et de santé chez les personnes présentant des symptômes compatibles avec un TAG seulement, un TAG et un EDM en comorbidité et un EDM seulement, population des ménages de 15 ans et plus, Canada (à l'exclusion des territoires), 2012

\begin{tabular}{|c|c|c|c|c|}
\hline \multirow{2}{*}{$\begin{array}{c}\text { Caractéristiques } \\
\text { sociodémographiques } \\
\text { et de santé }\end{array}$} & $\begin{array}{l}\text { TAG seulement } \\
(n=346 ; 1,2 \%)\end{array}$ & $\begin{array}{c}\text { TAG et EDM } \\
(\mathrm{n}=358 ; 1,3 \%)\end{array}$ & $\begin{array}{l}\text { EDM seulement } \\
(\mathrm{n}=894 ; 3,4 \%)\end{array}$ & \multirow[t]{2}{*}{ Valeur $p$} \\
\hline & $\%$ (IC à $95 \%)$ & $\%$ (IC à $95 \%)$ & \% (IC à $95 \%)$ & \\
\hline \multicolumn{5}{|l|}{ Sexe } \\
\hline Femmes & $60,2 \quad(52,6$ à 67,7$)$ & $68,2 \quad(60,7$ à 75,7$)$ & $61,2 \quad(56,2$ à 66,3$)$ & 0,23 \\
\hline \multicolumn{5}{|l|}{ Âge } \\
\hline $\begin{array}{l}\text { Âge moyen (ans) } \\
\text { Tranches d'âge (ans) }\end{array}$ & $43,8 \quad(41,2$ à 46,5$)$ & $42,2(40,0$ à 44,4$)$ & $38,0 \quad(36,5$ à 39,5) & $<0,001$ \\
\hline 15 à 29 & $22,8 \quad(17,2$ à 28,4$)$ & $22,7 \quad(15,9$ à 29,5$)$ & 37,4 (32,2 à 42,6) & $<0,001$ \\
\hline 30 à 49 & $37,9 \quad(30,8$ à 45,1$)$ & $48,6 \quad(40,5$ à 56,6$)$ & $39,6 \quad(34,1$ à 45,2$)$ & \\
\hline 50 et plus & $39,3 \quad(31,6$ à 47,0) & 28,8 (22,3 à 35,3) & $23,0 \quad(19,3$ à 26,7$)$ & \\
\hline
\end{tabular}

État matrimonial

Célibataire

$30,8 \quad(23,9$ à 37,7$) \quad 32,4 \quad(25,3$ à 39,4$) \quad 43,4 \quad(38,1$ à 48,6$)$

0,05

Divorcé/séparé/veuf

$20,8 \quad(14,0$ à 27,6) $\quad 19,7 \quad(14,1$ à 25,4) $\quad 16,6 \quad(11,9$ à 21,2)

Marié/conjoint de fait

$48,4 \quad(40,3$ à 56,6) $\quad 47,9 \quad(39,7$ à 56,1 $) \quad 40,1 \quad(34,9$ à 45,3)

Niveau de scolarité (répondant)

Études secondaires partielles

$15,0^{\mathrm{E}}(9,2$ à 20,9$) \quad 18,2 \quad(12,9$ à 23,6$) \quad 18,2 \quad(14,3$ à 22,1$)$

0,50

Diplôme d'études secondaires

$15,3^{\mathrm{E}}(9,1$ à 21,4$) \quad 14,8^{\mathrm{E}}(9,4$ à 20,3$) \quad 16,1 \quad(12,6$ à 19,6$)$

Études postsecondaires partielles

$6,5^{\mathrm{E}}(3,3$ à 9,7$) \quad 11,7^{\mathrm{E}}(5,7$ à 17,8$) \quad 11,8 \quad(8,3$ à 15,3$)$

Diplôme d'études postsecondaires

$63,2 \quad(55,5$ à 70,9) $\quad 55,2 \quad(47,6$ à 62,8$) \quad 53,9 \quad(48,6$ à 59,2) 
TABLEAU 1 (suite)

Caractéristiques sociodémographiques et de santé chez les personnes présentant des symptômes compatibles avec un TAG seulement, un TAG et un EDM en comorbidité et un EDM seulement, population des ménages de 15 ans et plus, Canada (à l'exclusion des territoires), 2012

\begin{tabular}{|c|c|c|c|c|}
\hline \multirow{2}{*}{$\begin{array}{l}\text { Caractéristiques } \\
\text { sociodémographiques } \\
\text { et de santé }\end{array}$} & $\begin{array}{c}\text { TAG seulement } \\
(\mathrm{n}=346 ; 1,2 \%)\end{array}$ & $\begin{array}{c}\text { TAG et EDM } \\
(\mathrm{n}=\mathbf{3 5 8 ;} \mathbf{1 , 3} \%)\end{array}$ & $\begin{array}{l}\text { EDM seulement } \\
(\mathrm{n}=894 ; 3,4 \%)\end{array}$ & \multirow[t]{2}{*}{ Valeur $p$} \\
\hline & \% (IC à $95 \%)$ & $\%$ (IC à $95 \%)$ & \% (IC à $95 \%)$ & \\
\hline \multicolumn{5}{|l|}{ Quintile de revenu (ménage) } \\
\hline $1^{\text {er }}$ & $22,8 \quad(17,0$ à 28,6$)$ & $36,6 \quad(28,5$ à 44,6$)$ & $32,0 \quad(26,8$ à 37,2$)$ & \multirow[t]{5}{*}{0,07} \\
\hline $2^{e}$ & $21,6 \quad(15,6$ à 27,7$)$ & $25,5 \quad(18,6$ à 32,3$)$ & $21,9 \quad(17,2$ à 26,6$)$ & \\
\hline $3^{e}$ & $21,3^{\mathrm{E}}(14,0$ à 28,7$)$ & $19,3^{\mathrm{E}}(12,8$ à 25,9$)$ & $17,0 \quad(13,3$ à 20,6$)$ & \\
\hline $4^{e}$ & $18,2^{\mathrm{E}}(11,7$ à 24,8$)$ & $10,2^{\mathrm{E}}(6,1$ à 14,3$)$ & $17,0 \quad(13,2$ à 20,8$)$ & \\
\hline $5^{e}$ & $16,0^{\mathrm{E}}(10,0$ à 21,9$)$ & $8,5^{\mathrm{E}}(4,5$ à 12,4$)$ & $12,1 \quad(9,0$ à 15,2$)$ & \\
\hline \multicolumn{5}{|l|}{ Activité professionnelle } \\
\hline Étudiant & $11,9^{\mathrm{E}}(7,9$ à 15,9$)$ & $10,7^{\mathrm{E}}(6,4$ à 15,0$)$ & $19,2 \quad(15,3$ à 23,2$)$ & \multirow[t]{3}{*}{0,03} \\
\hline Sans emploi & $45,1 \quad(37,3$ à 52,8) & $50,6 \quad(42,1$ à 59,1$)$ & $39,7 \quad(34,1$ à 45,2$)$ & \\
\hline En activité & $43,0 \quad(35,6$ à 50,4$)$ & $38,7 \quad(29,9$ à 47,5$)$ & $41,1 \quad(36,0$ à 46,2$)$ & \\
\hline \multicolumn{5}{|l|}{ Immigration } \\
\hline Immigrant & $16,3^{\mathrm{E}}(9,2$ à 23,4$)$ & $14,1^{\mathrm{E}}(7,7$ à 20,4$)$ & $17,9 \quad(13,4$ à 22,4$)$ & \multirow[t]{2}{*}{0,65} \\
\hline Non-immigrant & 83,7 (76,6 à 90,8) & $85,9 \quad(79,6$ à 92,3) & $82,1 \quad(77,6$ à 86,6) & \\
\hline \multicolumn{5}{|l|}{ Statut d'Autochtone } \\
\hline Autochtone & $5,6^{\mathrm{E}}(2,6$ à 8,6$)$ & 7,4E (3,3 à 11,4) & $6,2^{\mathrm{E}}(3,2$ à 9,2$)$ & \multirow[t]{2}{*}{0,80} \\
\hline Non-Autochtone & $94,4 \quad(91,4$ à 97,4) & $92,6 \quad(88,6$ à 96,7$)$ & $93,8 \quad(90,8$ à 96,8$)$ & \\
\hline \multicolumn{5}{|l|}{ Milieu } \\
\hline Rural & $18,3^{\mathrm{E}}(12,2$ à 24,3$)$ & $17,1 \quad(11,6$ à 22,5$)$ & $13,3 \quad(10,2$ à 16,4$)$ & \multirow[t]{2}{*}{0,19} \\
\hline Urbain & 81,8 (75,8 à 87,8) & $82,9 \quad(77,5$ à 88,4) & 86,7 (83,6 à 89,8) & \\
\hline \multicolumn{5}{|l|}{ Tabagisme } \\
\hline Fumeur quotidien & $22,0 \quad(15,9$ à 28,1$)$ & $33,0 \quad(25,8$ à 40,1$)$ & $26,0 \quad(21,5$ à 30,4$)$ & \multirow[t]{3}{*}{0,04} \\
\hline $\begin{array}{l}\text { Fumeur occasionnel } \\
\text { ou ex-fumeur }\end{array}$ & $33,7 \quad(26,7$ à 40,8) & $38,7 \quad(31,0$ à 46,4$)$ & $40,7 \quad(35,7$ à 45,6$)$ & \\
\hline Non-fumeur & 44,3 (36,1 à 52,4) & 28,4（20,6 à 36,1) & $33,4 \quad(27,7$ à 39,1) & \\
\hline \multicolumn{5}{|c|}{ Trouble de consommation de substances } \\
\hline Oui & $10,2^{\mathrm{E}}(6,1$ à 14,3$)$ & $17,6^{\mathrm{E}}(11,8$ à 23,3$)$ & $16,0 \quad(11,7$ à 20,2$)$ & \multirow[t]{2}{*}{0,12} \\
\hline Non & $89,8 \quad(85,7$ à 93,9) & $82,4 \quad(76,7$ à 88,2$)$ & $84,0 \quad(79,8$ à 88,3$)$ & \\
\hline \multicolumn{5}{|l|}{ Comorbidités physiques } \\
\hline 3 et plus & $18,1^{\mathrm{E}}(11,0$ à 25,3$)$ & $19,3 \quad(13,1$ à 25,5$)$ & 8,6 (6,1 à 11,1) & \multirow[t]{3}{*}{$<0,001$} \\
\hline 1 à 2 & 47,8 (39,9 à 55,8) & $49,2(41,3$ à 57,1$)$ & $40,4 \quad(35,3$ à 45,6$)$ & \\
\hline Aucune & $34,1 \quad(26,9$ à 41,2$)$ & $31,6(23,5$ à 39,7$)$ & $51,0 \quad(45,7$ à 56,2$)$ & \\
\hline
\end{tabular}

Abréviations : EDM, épisode dépressif majeur; IC, intervalle de confiance; TAG, trouble d’anxiété généralisée.

Remarques : Les $\mathbf{n}$ sont fondés sur des données non pondérées, alors que les proportions (\%), les moyennes et les IC à $95 \%$ sont fondés sur des données pondérées.

Ce tableau présente les données au cours des 12 derniers mois $(n=1598)$.

${ }^{E}$ Variabilité d'échantillonnage élevée (coefficient de variation entre 16,6 et 33,3\%).

comorbidité ont fait état d'un état de santé perçu sous-optimal, d'insatisfaction par rapport à leur vie et de détresse psychologique sévère, plus des deux tiers, d'un état de santé mentale sous-optimal et près du quart, d'une incapacité sévère ou extrême. La relation entre les sous-groupes étudiés et toutes les autres mesures de l'état de santé s'est révélée statistiquement significative (tableau 2).
Fait intéressant, moins de $60 \%$ des personnes présentant un TAG ou un EDM seulement ont consulté un professionnel de la santé, alors que près de $75 \%$ des personnes présentant un TAG et un EDM en comorbidité ont mentionné l'avoir fait. De même, les personnes présentant un TAG et un EDM en comorbidité ont été plus nombreuses à consulter un professionnel de la santé mentale (40 \%) que les personnes présentant un EDM seulement (environ $30 \%$ ) ou un TAG seulement (près de $25 \%$ ).

Questionnées à propos de la satisfaction de leurs besoins en soins de santé mentale, environ $30 \%$ des personnes présentant un TAG ou un EDM seulement ont répondu que leurs besoins avaient été satisfaits partiellement ou n'avaient pas été satisfaits, cette proportion passant à près de $50 \%$ chez les personnes présentant un TAG et un EDM en comorbidité. Les relations entre les sous-groupes étudiés et les consultations médicales ou le besoin perçu en soins étaient toutes statistiquement significatives (tableau 3).

Après ajustement en fonction de toutes les caractéristiques sociodémographiques, comportementales et de santé, toutes les mesures de l'état de santé se sont avérées similaires (ou inférieures, dans le cas des incapacités modérées) chez les personnes présentant un TAG seulement à celles rapportées par les personnes présentant un EDM seulement (tableau 4). En revanche, les personnes présentant un TAG et un DEM en comorbidité étaient environ 2,5 fois plus nombreuses à avoir fait état d'un état de santé mentale passable ou mauvais, 8 fois plus nombreuses à avoir fait état de détresse psychologique sévère et près de 10 fois plus nombreuses à avoir vécu une incapacité extrême que les personnes présentant un EDM seulement. Elles étaient aussi 2 fois plus nombreuses à avoir mentionné que leurs besoins en matière de soins avaient été satisfaits partiellement ou n'avaient pas été satisfaits.

Nous n'avons observé aucun effet significatif lié à l'âge (données non présentées).

Comme nous nous y attendions, les personnes présentant un TAG (en comorbidité ou non avec un EDM) ont obtenu des résultats statistiquement inférieurs à celles exemptes de TAG et d'EDM sur le plan de l'état de santé perçu, de la santé mentale autoévaluée, de la détresse psychologique et de l'incapacité. Les personnes présentant un 
TABLEAU 2

Facteurs liés à l'état de santé chez les personnes présentant des symptômes compatibles avec un TAG seulement, un TAG et un EDM en comorbidité et un EDM seulement, population des ménages de 15 ans et plus, Canada (à l'exclusion des territoires), 2012

\begin{tabular}{|c|c|c|c|c|}
\hline \multirow[t]{2}{*}{ Facteurs liés à l'état de santé } & $\begin{array}{l}\text { TAG seulement } \\
(\mathrm{n}=346 ; 1,2 \%)\end{array}$ & $\begin{array}{c}\text { TAG et EDM } \\
(n=358 ; 1,3 \%)\end{array}$ & $\begin{array}{l}\text { EDM seulement } \\
(\mathrm{n}=894 ; 3,4 \%)\end{array}$ & \multirow[t]{2}{*}{ Valeur $p$} \\
\hline & \% (IC à $95 \%)$ & \% (IC à $95 \%)$ & \% (IC à $95 \%)$ & \\
\hline \multicolumn{5}{|l|}{ État de santé perçu } \\
\hline Passable ou mauvais & 29,7 (22,2 à 37,2) & $47,3 \quad(39,4$ à 55,1$)$ & 24,7 (20,2 à 29,2) & $<0,001$ \\
\hline \multicolumn{5}{|c|}{ État de santé mentale autodéclaré } \\
\hline Passable ou mauvais & $44,7 \quad$ (36,8 à 52,6) & $69,9(62,8$ à 77,0$)$ & 45,3 (40,1 à 50,5) & $<0,001$ \\
\hline \multicolumn{5}{|c|}{ Détresse psychologique dans le dernier mois } \\
\hline Sévère & $17,8^{\mathrm{E}}(11,4$ à 24,2$)$ & $51,1 \quad(43,5$ à 58,7$)$ & $19,4(15,0$ à 23,7$)$ & \multirow[t]{3}{*}{$<0,001$} \\
\hline Modérée & $63,4 \quad(55,4$ à 71,4$)$ & $42,9 \quad(35,1$ à 50,7$)$ & $59,4(54,1$ à 64,7$)$ & \\
\hline Aucune & $18,8^{\mathrm{E}}(12,3$ à 25,4$)$ & $6,0^{\mathrm{E}}(2,9$ à 9,1$)$ & $21,2(17,3$ à 25,2) & \\
\hline \multicolumn{5}{|c|}{ Incapacité dans les 30 derniers jours } \\
\hline Sévère ou extrême & n.d. & $22,2 \quad(15,6$ à 28,8$)$ & $6,0(4,2$ à 7,9$)$ & \multirow[t]{4}{*}{$<0,001$} \\
\hline Modérée & $28,1 \quad(19,5$ à 36,7) & $30,2 \quad(22,7$ à 37,6) & $18,8(15,0$ à 22,5$)$ & \\
\hline Légère & $47,8 \quad(39,2$ à 56,4$)$ & $37,5 \quad(29,4$ à 45,5$)$ & 43,4 (38,0 à 48,8) & \\
\hline Aucune & $19,3 \quad(13,2$ à 25,5$)$ & $10,2^{\mathrm{E}}(5,5$ à 14,9$)$ & $31,8(26,8$ à 36,9) & \\
\hline
\end{tabular}

Abréviations : EDM, épisode dépressif majeur; IC, intervalle de confiance; n.d., non disponible (coefficient de variation supérieu à 33,3); TAG, trouble d'anxiété généralisée.

Remarques : Les $\mathbf{n}$ sont fondés sur des données non pondérées, alors que les proportions (\%) et les IC à $95 \%$ sont fondés sur des données pondérées.

Ce tableau présente les données au cours des 12 derniers mois $(n=1598)$.

E Variabilité d'échantillonnage élevée (coefficient de variation entre 16,6 et 33,3 \%).

TAG (en comorbidité ou non avec un EDM) étaient aussi plus nombreuses à avoir consulté un professionnel de la santé pour des symptômes liés à la santé mentale, et leur

besoin perçu de soins de santé mentale était plus grand que celui des personnes sans TAG et EDM (données non présentées, disponibles sur demande auprès des auteurs).

TABLEAU 3

Consultations médicales et besoins perçus en soins de santé chez les personnes présentant des symptômes compatibles avec un TAG seulement, un TAG et un EDM en comorbidité et un EDM seulement, population des ménages de 15 ans et plus, Canada (à l'exclusion des territoires), 2012

\begin{tabular}{|c|c|c|c|c|}
\hline \multirow{2}{*}{$\begin{array}{l}\text { Consultations } \\
\text { et besoins perçus }\end{array}$} & $\begin{array}{l}\text { TAG seulement } \\
(\mathrm{n}=346 ; 1,2 \%)\end{array}$ & $\begin{array}{c}\text { TAG et EDM } \\
(n=358 ; 1,3 \%)\end{array}$ & $\begin{array}{l}\text { EDM seulement } \\
(\mathrm{n}=894 ; 3,4 \%)\end{array}$ & \multirow[t]{2}{*}{ Valeur $p$} \\
\hline & \% (IC à $95 \%)$ & \% (IC à $95 \%)$ & \% (IC à $95 \%)$ & \\
\hline \multicolumn{5}{|c|}{ Consultations médicales dans les 12 derniers mois } \\
\hline Oui & $56,2(48,7-63,6)$ & $72,7 \quad(64,9-80,4)$ & $59,9(54,8-65,1)$ & 0,008 \\
\hline \multicolumn{5}{|c|}{ Consultations en santé mentale dans les 12 derniers mois } \\
\hline Oui & $33,0(26,0-40,0)$ & $52,6 \quad(44,4-60,9)$ & $42,7 \quad(37,3-48,1)$ & 0,003 \\
\hline \multicolumn{5}{|c|}{ Besoins perçus en soins de santé dans les 12 derniers mois } \\
\hline Aucun & $26,0(18,4-33,7)$ & $8,6^{\mathrm{E}}(3,6-13,5)$ & $22,5(18,5-26,6)$ & $<0,001$ \\
\hline Satisfaits & $43,3(34,7-51,9)$ & $42,9 \quad(34,3-51,6)$ & $45,7 \quad(40,2-51,3)$ & \\
\hline $\begin{array}{l}\text { Partiellement } \\
\text { ou non satisfaits }\end{array}$ & $30,7(23,4-37,9)$ & $48,5 \quad(40,3-56,8)$ & $31,8(26,9-36,6)$ & \\
\hline
\end{tabular}

Abréviations : EDM, épisode dépressif majeur; IC, intervalle de confiance; TAG, trouble d’anxiété généralisée.

Remarques : Les $\mathbf{n}$ sont fondés sur des données non pondérées, alors que les proportions (\%) et les IC à $95 \%$ sont fondés sur des données pondérées.

Ce tableau présente les données au cours des 12 derniers mois $(n=1598)$.

${ }^{E}$ Variabilité d'échantillonnage élevée (coefficient de variation entre 16,6 et 33,3\%).

\section{Analyse}

En 2012, environ 700000 (2,5\%) Canadiens de 15 ans et plus présentaient des symptômes compatibles avec un TAG. La moitié d'entre eux présentaient également des symptômes d'EDM. Ces estimations pourraient être conservatrices, étant donné que les personnes affectées par un TAG (et un EDM) évaluent négativement leur santé et leur santé mentale et qu'il a été démontré que les non-répondants sont nettement plus nombreux à faire une évaluation négative de leur santé, même après ajustement en fonction du sexe, de l'âge, du pays de naissance et du niveau de scolarité29. Toutefois, à notre connaissance, la question du biais de non-réponse chez les personnes atteintes de différents types de troubles mentaux, tels que le TAG et l'EDM, n'a pas encore été étudiée.

Cette étude met en relief le fait que les personnes présentant un TAG seulement ont obtenu des résultats équivalents (ou légèrement moins bons) à ceux des personnes présentant un EDM seulement sur le plan de l'état de santé perçu, de l'état de santé mentale autodéclaré, de la détresse psychologique et de l'incapacité, comme il est fait mention ailleurs $\mathrm{s}^{3,7,16,30}$.

Notre étude a aussi fait ressortir de moins bons résultats de santé chez les personnes présentant un TAG et un EDM en comorbidité, ainsi qu'une grande limitation des activités de la vie quotidienne associée à ces troubles, qui sont responsables d'un grand nombre d'incapacités. Ces observations concordent avec celles d'autres études sur l'anxiété et la dépression en comorbidité3,7,12,31-35. Les professionnels de la santé qui reçoivent des personnes présentant des symptômes d'anxiété ou de dépression devraient donc vérifier soigneusement la présence d'affections psychologiques en comorbidité. De plus, comme près de $70 \%$ des personnes présentant un TAG et un EDM en comorbidité avaient au moins un problème de santé physique chronique, et à la lumière des données solides prouvant l'existence d'une relation bidirectionnelle entre les troubles mentaux (plus spécifiquement, la dépression et l'anxiété) et les résultats de santé physique ${ }^{36}$, une attention particulière devrait être accordée à la prévention et à la prise en charge des problèmes de santé physique chroniques comorbides. 
TABLEAU 4

Rapport de cotes ajusté de la présence de symptômes compatibles avec un TAG seulement ou un TAG et EDM en comorbidité par rapport à la présence de symptômes compatibles avec un EDM seulement en fonction de l'état de santé, de la consultation d'un professionnel de la santé et des besoins perçus de soins de santé, population des ménages de 15 ans et plus, Canada (à l'exclusion des territoires), 2012

\begin{tabular}{|c|c|c|}
\hline & & RC (IC à $95 \%)$ \\
\hline \multicolumn{3}{|l|}{ État de santé perçu } \\
\hline \multirow{3}{*}{$\begin{array}{l}\text { Passable ou mauvais vs excellent, très } \\
\text { bon ou bon }\end{array}$} & TAG seulement & $1,2(0,7$ à 2,0$)$ \\
\hline & TAG et EDM & $1,9(1,1$ à 3,3$)$ \\
\hline & EDM seulement & Population de référence \\
\hline \multicolumn{3}{|l|}{ État de santé mentale autodéclaré } \\
\hline \multirow{3}{*}{$\begin{array}{l}\text { Passable ou mauvais vs excellent, très } \\
\text { bon ou bon }\end{array}$} & TAG seulement & $1,2(0,8$ à 1,8$)$ \\
\hline & TAG et EDM & $2,6(1,6$ à 4,1$)$ \\
\hline & EDM seulement & Population de référence \\
\hline \multicolumn{3}{|c|}{ Détresse psychologique durant le dernier mois } \\
\hline \multirow{3}{*}{ Sévère vs aucune } & TAG seulement & $1,8(0,9$ à 3,6) \\
\hline & TAG et EDM & $7,9(3,5$ à 17,5$)$ \\
\hline & EDM seulement & Population de référence \\
\hline \multirow{3}{*}{ Modérée vs aucune } & TAG seulement & $1,8(1,0$ à 3,0$)$ \\
\hline & TAG et EDM & $2,4(1,2$ à 5,0$)$ \\
\hline & EDM seulement & Population de référence \\
\hline \multicolumn{3}{|l|}{ Incapacité durant les 30 derniers jours } \\
\hline \multirow{3}{*}{ Sévère ou extrême vs aucune } & TAG seulement & $1,4(0,5$ à 4,1$)$ \\
\hline & TAG et EDM & $9,8(4,0$ à 23,9$)$ \\
\hline & EDM seulement & Population de référence \\
\hline \multirow{3}{*}{ Modérée vs aucune } & TAG seulement & $2,3(1,3$ à 4,3$)$ \\
\hline & TAG et EDM & 4,9 (2,3 à 10,4) \\
\hline & EDM seulement & Population de référence \\
\hline \multirow{3}{*}{ Légère vs aucune } & TAG seulement & $1,8(1,0$ à 3,1$)$ \\
\hline & TAG et EDM & $2,9(1,4$ à 5,7$)$ \\
\hline & EDM seulement & Population de référence \\
\hline \multicolumn{3}{|c|}{ Consultations médicales durant les 12 derniers mois } \\
\hline \multirow[t]{3}{*}{ Oui ou non } & TAG seulement & $0,8(0,6$ à 1,3$)$ \\
\hline & TAG et EDM & $1,6(1,0$ à 2,6$)$ \\
\hline & EDM seulement & Population de référence \\
\hline \multicolumn{3}{|c|}{ Consultations en santé mentale durant les 12 derniers mois } \\
\hline \multirow{3}{*}{ Oui ou non } & TAG seulement & $0,8(0,5$ à 1,2$)$ \\
\hline & TAG et EDM & $1,4(0,9$ à 2,3$)$ \\
\hline & EDM seulement & Population de référence \\
\hline \multicolumn{3}{|l|}{ Besoins perçus de soins de santé } \\
\hline \multirow{3}{*}{$\begin{array}{l}\text { Partiellement ou non satisfaits vs tous } \\
\text { satisfaits }\end{array}$} & TAG seulement & $1,4(0,8$ à 2,3) \\
\hline & TAG et EDM & $1,8(1,1$ à 3,0$)$ \\
\hline & EDM seulement & Population de référence \\
\hline
\end{tabular}

Abréviations : EDM, épisode dépressif majeur; IC, intervalle de confiance; RC, rapport de cotes; TAG, trouble d’anxiété généralsée; vs, versus.

Remarques : Les RC et les IC à $95 \%$ sont ajustés en fonction du sexe, de l'âge, de l'état matrimonial, du niveau de scolarité (répondant), du quintile de revenu (ménage), de l'activité professionnelle, du statut vis-à-vis de l'immigration, du statut d'Autochtone, du milieu de résidence, du tabagisme, des troubles de consommation de substances ainsi que des comorbidités physiques. Ils sont fondés sur des données pondérées.

Ce tableau présente les données au cours des 12 derniers mois $(n=1598)$.
Notre étude révèle que près de $50 \%$ des personnes présentant un TAG et un EDM en comorbidité jugeaient que leurs besoins en soins de santé mentale avaient été partiellement satisfaits ou n'avaient pas été satisfaits, contre environ $30 \%$ des personnes présentant un TAG ou un EDM seulement. Bien que nous ne puissions pas estimer ici la proportion de personnes présentant un TAG ayant reçu un diagnostic et un traitement adéquats, d'autres études ont montré que le TAG est généralement mal reconnu ${ }^{4,16}$ et que les deux tiers des patients atteints d'un trouble d'anxiété ne reçoivent pas de traitement fondé sur des données probantes ${ }^{30,33,37}$.

\section{Points forts et limites}

Notre étude comporte plusieurs points forts, notamment son échantillon de grande taille représentatif de la population et le fait que l'enquête ait été administrée par des professionnels qualifiés, selon un format structuré.

Cependant, nos observations doivent être interprétées en tenant compte de plusieurs limites importantes. D’abord, les résultats sont fondés sur des données autodéclarées, qui sont donc sujettes aux biais de désirabilité sociale, de rappel et de non-déclaration volontaire. Ensuite, le taux de réponse relativement faible à l'enquête $(68,9 \%)$ est préoccupant. On peut supposer que les personnes affectées par des troubles de l'humeur et des troubles d'anxiété, particulièrement celles souffrant de symptômes sévères et d'une perception négative de leur état de santé29, sont plus réticentes ou hésitantes à participer à l'enquête. Nos résultats sous-estiment donc sans doute la prévalence réelle de ces affections et ils sont vraisemblablement influencés par les répondants ayant participé, ce qui constituerait une forme de biais de participation. Bien que les méthodes d'ajustement de la pondération de Statistique Canada aident à réduire l'incidence de la non-participation en général, il est possible qu'elles n'aient pas prévenu le biais de non-participation dans cette étude. Par ailleurs, les résultats concernant le type d'affection sont fondés sur les critères du questionnaire CIDI de l'Organisation mondiale de la santé, et non sur des évaluations cliniques par des professionnels de la santé mentale. Enfin, les résultats ont été obtenus à l'aide d'un plan transversal, ce qui ne permet pas de déterminer si les facteurs connexes ont 
contribué à l'apparition du TAG ou de l'EDM ou s'ils en résultent.

\section{Conclusion}

Le TAG est une affection courante fréquemment accompagnée d'un EDM. Il peut profondément bouleverser la vie des personnes qui en sont atteintes. Bien que les personnes avec un TAG soient comparables à celles avec un EDM sur le plan de l'état de santé perçu, de la détresse psychologique et de l'incapacité, celles présentant ces deux affections en comorbidité ont de moins bons résultats de santé. Comme dans le cas de la dépression, des mesures d'amélioration de la reconnaissance et de la prise en charge du TAG, ainsi que du TAG en comorbidité avec un EDM, sont nécessaires pour réduire la sévérité et la persistance des symptômes et pour éviter d'autres problèmes de santé mentale ou des maladies physiques chroniques.

\section{Remerciements}

Cette recherche n'a bénéficié d'aucun financement d'organisme subventionnaire, du secteur commercial ou d'organisme sans but lucratif.

\section{Conflits d'intérêts}

Aucun.

\section{Contribution des auteurs}

Tous les auteurs ont contribué à la conceptualisation de l'étude, SO a analysé les données et LP a rédigé le manuscrit. Tous les auteurs ont contribué à l'interprétation des données et ont fourni des commentaires sur l'ébauche du manuscrit.

\section{Références}

1. Pearson C, Janz T, Ali J. Coup d'œil sur la santé : troubles mentaux et troubles liés à l'utilisation de substances au Canada. Ottawa (Ont.) : Statistique Canada; 2013. [nº 82-624-X]

2. Kessler RC, Chiu WT, Demler O, Walters EE. Prevalence, severity, and comorbidity of 12-month DSM-IV disorders in the National Comorbidity Survey Replication. Arch Gen Psychiatry. 2005;62(6):617-627.
3. Hunt C, Issakidis C, Andrews G. DSM-IV generalized anxiety disorder in the Australian National Survey of Mental Health and Well-Being. Psychol Med. 2002;32(4):649-659.

4. Lieb R, Becker E, Altamura C. The epidemiology of generalized anxiety disorder in Europe. Eur Neuropsychopharmacol. 2005;15(4): 445-452.

5. Hoge EA, Ivkovic A, Fricchione GL. Generalized anxiety disorder: diagnosis and treatment. BMJ. 2012;345:e7500.

6. American Psychiatric Association. Diagnostic and statistical manual of mental disorders ( $5^{\mathrm{e}}$ éd.). Arlington (VA) : American Psychiatric Publishing; 2013.

7. Kessler RC, Keller MB, Wittchen HU. The epidemiology of generalized anxiety disorder. Psychiatr Clin North Am. 2001;24(1):19-39.

8. Bruce SE, Yonkers KA, Otto MW, et al. Influence of psychiatric comorbidity on recovery and recurrence in generalized anxiety disorder, social phobia, and panic disorder: a 12-year prospective study. Am J Psychiatry. 2005; 162(6):1179-1187.

9. Angst J, Gamma A, Baldwin DS, Ajdacic-Gross V, Rössler W. The generalized anxiety spectrum: prevalence, onset, course and outcome. Eur Arch of Psychiatry Clin Neurosci. 2009; 259(1):37-45.

10. Ramsawh HJ, Weisberg RB, Dyck I, Stout R, Keller MB. Age of onset, clinical characteristics, and 15-year course of anxiety disorders in a prospective, longitudinal, observational study. J Affect Disord. 2011;132(1-2):260-264.

11. Kisely S, Scott A, Denney J, Simon G. Duration of untreated symptoms in common mental disorders: association with outcomes: international study. $\mathrm{Br}$ J Psychiatry. 2006;189:79-80.

12. Nutt D, Argyropoulos S, Hood S, Potokar J. Generalized anxiety disorder: a comorbid disease. Eur Neuropsychopharmacol. 2006; 16(Suppl 2):S109-S118.
13. Allgulander C. Generalized anxiety disorder: a review of recent findings. $\mathrm{J}$ Experiment Clin Med. 2012;4(2):88-91.

14. Hettema JM. The nosologic relationship between generalized anxiety disorder and major depression. Depress Anxiety. 2008;25(4):300-316.

15. Ormel J, Petukhova M, Chatterji S, et al. Disability and treatment of specific mental and physical disorders across the world. Br J Psychiatry. 2008; 192(5):368-375.

16. Wittchen, HU. Generalized anxiety disorder: prevalence, burden, and cost to society. Depress Anxiety. 2002;16(4): 162-171.

17. Bélanger L, Ladouceur R, Morin CM. Generalized anxiety disorder and health care use. Can Fam Physician. 2005;51:1362-1363.

18. Statistique Canada. Enquête sur la santé dans les collectivités canadiennes (ESCC) - Santé mentale Guide de l'utilisateur. Ottawa (Ont.) : Statistique Canada; 2013.

19. Kessler RC, Üstün TB. The World Mental Health (WMH) Survey Initiative Version of the World Health Organization (WHO) Composite International Diagnostic Interview (CIDI). Int J Methods Psychiatr Res. 2004; 13(2):93-121.

20. Kessler RC, Calabrese JR, Farley PA, et al. Composite International Diagnostic Interview screening scales for DSM-IV anxiety and mood disorders. Psychol Med. 2013;43(8):1625-1637.

21. Statistique Canada. Enquête sur la santé dans les collectivités canadiennes - Santé mentale (ESCC). Spécifications des variables dérivées (VD) [Internet]. Ottawa (Ont.) : Statistique Canada; 2013. En ligne à : http:// odesi2.scholarsportal.info/webview /index/en/Odesi/ODESI-Click-to -View-Categories-.d.6/-Sant-.d.42 /CANADA.d.43/Enqu-te-sur-la-sant -dans-les-collectivit-s-canadiennes -ESCC-.d.331/2012.d.1358/Enqu-te -sur-la-sant-dans-les-collectivit-s -canadiennes-2012-Sant-mentale/ fStudy/escc-82M0013-F-2012-sante -mentale 
22. Kessler RC, Barker PR, Colpe LJ, et al. Screening for serious mental illness in the general population Arch Gen Psychiatry. 2003;60(2):184-189.

23. Prochaska JJ, Sung HY, Max W, Shi Y, Ong M. Validity study of the K6 scale as a measure of moderate mental distress based on mental health treatment need and utilization. Int $\mathrm{J}$ Methods Psychiatr Res. 2012;21(2); 88-97.

24. Üstün TB, Kostanjsek, N, Chatterji S, Rehm J. (dir.). Measuring health and disability: manual for WHO Disability Assessment Schedule (WHODAS 2.0). Genève $(\mathrm{CH})$ : Organisation mondiale de la santé; 2010. En ligne à : http:// whqlibdoc.who.int/publications /2010/9789241547598_eng.pdf

25. Virués-Ortega J, de Pedro-Cuesta J, Seijo-Martínez M, et al. Prevalence of disability in a composite $\geq 75$-year-old population in Spain: a screening survey based on the International Classification of Functioning. BMC Public Health. 2011;11:176.

26. Rust K, Rao JNK. Variance estimation for complex surveys using replication techniques. Stat Methods Med Res. 1996;5(3):281-310.

27. Mawani FN, Gilmour H. Validation of self-rated mental health. Health Rep. 2010;21(3):1-15.

28. Toghanian S, Di Bonaventura M, Järbrink K, Locklear JC. Economic and humanistic burden of illness in generalized anxiety disorder: an analysis of patient survey data in Europe. Clinicoecon Outcomes Res. 2014;6(1): 151-163.

29. Lindén-Boström M, Persson C. A selective follow-up study on a public health survey. Eur J Public Health. 2013;23(1):152-157.

30. Hoffman DL, Dukes EM, Wittchen HU. Human and economic burden of generalized anxiety disorder. Depress Anxiety. 2008;25(1):72-90.

31. McEvoy PM, Grove R, Slade T. Epidemiology of anxiety disorders in the Australian general population: findings of the 2007 Australian National Survey of Mental Health and Wellbeing. Aust N Z J Psychiatry. 2011; 45(11):957-967.
32. Leray E, Camara A, Drapier D, et al. Prevalence, characteristics and comorbidities of anxiety disorders in France: results from the "Mental Health in General Population” survey (MHGP). Eur Psychiatry. 2011;26(6):339-345.

33. Kroenke K, Spitzer RL, Williams JB, Monahan PO, Löwe B. Anxiety disorders in primary care: prevalence, impairment, comorbidity, and detection. Ann Intern Med. 2007;146(5): 317-325.

34. Belzer K, Schneier FR. Comorbidity of anxiety and depressive disorders: issues in conceptualization, assessment, and treatment. J Psychiatr Pract. 2004;10(5):296-306.

35. Grant BF, Hasin DS, Stinson FS, et al. Prevalence, correlates, co-morbidity, and comparative disability of DSM-IV generalized anxiety disorder in the USA: results from the National Epidemiologic Survey on Alcohol and Related Conditions. Psychol Med. 2005;35(12):1747-1759.

36. Kolappa K, Henderson DC, Kishore SP No physical health without mental health: lessons unlearned? Bull World Health Organ. 2013;91(1):3-3A.

37. Culpepper, L. Generalized anxiety disorder in primary care: emerging issues in management and treatment. J Clin Psychiatry. 2002;63:35-42. 